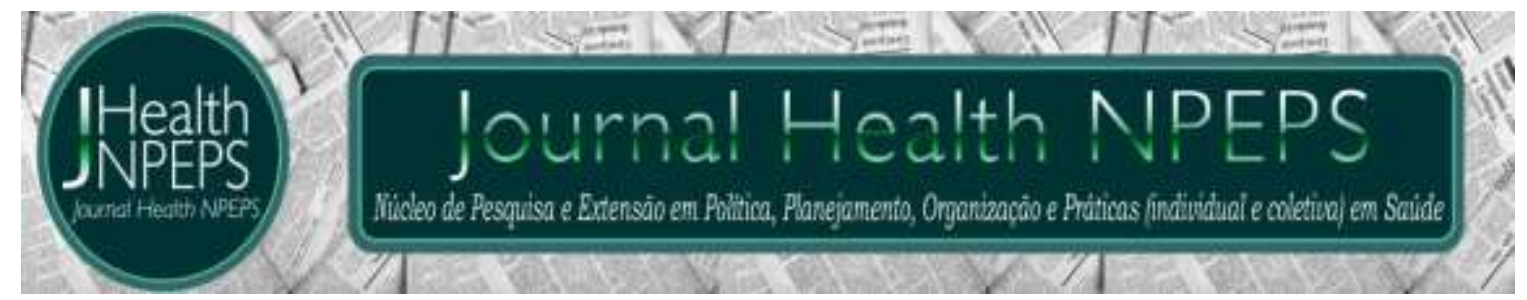

http://dx.doi.org/10.30681/252610104184

ARTIGO ORIGINAL

\title{
Conhecimentos, atitudes e práticas de mulheres frente ao exame preventivo do câncer do colo uterino
}

\author{
Knowledge, attitudes and practices of women in the face of preventive \\ cervical cancer test
}

\section{Conocimiento, actitudes y practicas de mujeres en la cara de la prueba de cáncer cervical preventivo}

\section{Anthagoras Dantas de Mesquita ${ }^{1}$, Karla Katrine Neri Teles ${ }^{2}$, Suelem Cristina Barroso da Silva ${ }^{3}$, Frankllin Ramon da Silva ${ }^{4}$, Leila Keury Costa Lima ${ }^{5}$, Ruth Silva Lima da Costa ${ }^{6}$, Eder Ferreira de Arruda ${ }^{7}$}

\section{RESUMO}

Objetivo: identificar os conhecimentos, atitudes e práticas de mulheres frente ao exame preventivo do câncer do colo uterino. Método: trata-se de um estudo transversal, desenvolvido junto a 160 mulheres atendidas em uma unidade de saúde do Acre. Os dados foram coletados através de entrevista e analisados a partir do programa Statical Package for the Sciences. Resultados: a maioria das mulheres estavam na faixa etária de 30 a 40 anos, com ensino médio completo, casadas, renda familiar de um salário mínimo, não faziam uso de tabaco ou álcool, com início da vida sexual após os 15 anos

\footnotetext{
${ }^{1}$ Enfermeiro. Especialista em Saúde da Família. Enfermeiro da Secretaria Municipal de Saúde. Rio Branco, Acre, Brasil. Email: anthagoras@hotmail.com ORCID ID: https://orcid.org/0000-0002-1220-302X Autor principal - Endereço para correspondência: Av. Brasil, no 475 - $2^{\circ}$ andar - Centro, Rio Branco - AC, 69900-078.

${ }^{2}$ Enfermeira. Graduada em Enfermagem pelo Centro Universitário Uninorte. Rio Branco, Acre, Brasil Email: Karlaneri349@gmail.com. ORCID ID: https://orcid.org/ 0000-0001-5310-3052

${ }^{3}$ Enfermeira. Graduada em Enfermagem pelo Centro Universitário Uninorte Rio Branco, Acre, Brasil Email: suelemcristinabarroso@gamil.com. ORCID ID: https://orcid.org/ 0000-0001-9577-2529

${ }^{4}$ Acadêmico de Enfermagem do Centro Universitário Uninorte. Rio Branco, Acre, Brasil. Email: frankllinramondasilva@gmail.com ORCID ID: https://orcid.org/0000-0002-3096-7373

${ }^{5}$ Acadêmica de Enfermagem do Centro Universitário Uninorte Rio Branco, Acre, Brasil. Email: leilakeury@hotmail.com ORCID ID: https://orcid.org/ 0000-0002-5283-7895

${ }^{6}$ Enfermeira. Mestre em Ciências da Saúde. Enfermeira Auditora na Secretaria de Estado de Saúde do Acre e Docente nos cursos de Medicina e Enfermagem do Centro Universitário Uninorte. Rio Branco, Acre, Brasil. Email: ruttylyma@gmail.com ORCID ID: https://orcid.org/ 0000-0003-1890-086X

${ }^{7}$ Biólogo e Médico Veterinário. Mestre em Saúde Coletiva. Docente dos cursos de Fisioterapia, Enfermagem e Biomedicina do Centro Universitário Uninorte. Rio Branco, Acre, Brasil. Email: ederarrud@gmail.com ORCID ID: https://orcid.org/0000-0002-9593-0029
} 
e sem histórico de infecção sexualmente transmissível. Entre as participantes, $53,1 \%$ demostraram ter conhecimento adequado sobre o câncer, bem como $97,5 \%$ e $58,8 \%$ possuindo atitudes e práticas adequadas, respectivamente. Conclusão: é essencial a continuidade das ações integradas de educação em saúde, a fim de prevenir e controlar a doença, bem como garantir a continuidade do acesso, adesão e conhecimento das mulheres sobre a importância do exame.

Descritores: Atenção Primária à Saúde; Diagnóstico; Saúde da Mulher.

\section{ABSTRACT}

Objective: to identify the knowledge, attitudes and practices of women regarding the cervical cancer preventive exam. Method: this is a crosssectional study, carried out with 160 women attended at a health unit in Acre. Data were collected through interviews and analyzed using the Statical Package for the Sciences program. Results: most women were in the age group of 30 to 40 years old, with complete high school education, married, family income of a minimum wage, did not use tobacco or alcohol, with sexual initiation after the age of 15 and no history of sexually transmitted infection. Among the participants, 53.1\% demonstrated having adequate knowledge about cancer, as well as $97.5 \%$ and $58.8 \%$ having appropriate attitudes and practices, respectively. Conclusion: it is essential to continue integrated health education actions in order to prevent and control the disease, as well as to guarantee the continuity of access, adherence and knowledge of women about the importance of the exam.

Descriptors: Primary Health Care; Diagnosis; Women's Health.

\section{RESUMEN}

Objetivo: identificar el conocimiento, las actitudes y las prácticas de las mujeres con respecto al examen preventivo del cáncer cervical. Método: este es un estudio transversal, realizado con 160 mujeres atendidas en una unidad de salud en Acre. Los datos fueron recolectados a través de entrevistas y analizados utilizando el paquete Statical Package for the Sciences. Resultados: la mayoría de las mujeres pertenecían al grupo de edad de 30 a 40 años, con educación secundaria completa, casadas, ingresos familiares con un salario mínimo, no consumían tabaco ni alcohol, con iniciación sexual después de 15 años y sin antecedentes de infección de transmisión sexual. Entre los participantes, el 53.1\% demostró tener un conocimiento adecuado sobre el cáncer, así como el $97.5 \%$ y $58.8 \%$ con actitudes y prácticas apropiadas, respectivamente. Conclusión: es esencial continuar con las acciones integradas de educación sanitaria para prevenir y controlar la enfermedad, así como para garantizar la continuidad del acceso, la adhesión y el conocimiento de las mujeres sobre la importancia del examen.

Descriptores: Atención Primaria de Salud; Diagnóstico; Salud de la Mujer.

\section{INTRODUÇÃO}


Conhecimentos, atitudes e práticas..

o câncer cervical é a terceira neoplasia mais incidente entre as mulheres brasileiras, sendo mais frequente entre aquelas de 50 e 60 anos. Esta neoplasia se desenvolve a partir de lesões primárias que não foram detectadas ou tratadas corretamente ${ }^{1}$.

Em 2018, estimou-se cerca de 570.000 casos e 311.000 mortes por este tipo de câncer em todo o mundo, configurando-se como a quarta principal causa de morte em mulheres ${ }^{2}$. No Brasil, houve uma previsão para o aparecimento de aproximadamente 16.370 novos casos entre os anos de 2018 e 2019, representando uma incidência de 15,43 casos para cada $100 \mathrm{mil}$ mulheres. Este câncer ocupa a primeira posição entre as neoplasias mais frequentes na Região Norte, com a incidência de 25,62 casos $^{3}$.

A principal medida utilizada na prevenção $\mathrm{e}$ identificação de lesões precursoras desta neoplasia é o exame citopatológico do colo do útero, mais conhecido como exame de Papanicolau. Ele se caracteriza por ser de simples realização e estar disponibilizado gratuitamente pelo Sistema Único de Saúde (SUS), através da cobertura de unidades de atenção primária em saúde, em todo território nacional ${ }^{4}$.

A Organização Mundial de Saúde (OMS) preconiza que a população alvo para a prática do exame são mulheres entre 25 e 64 anos, e que a cobertura dessa população deve ser de no mínimo $80 \%$, sendo que com o diagnóstico e - tratamento adequado pode-se reduzir em até $90 \%$ a chance de se desenvolver esse câncer de forma invasiva. A priorização dessa faixa etária como público alvo para a realização do exame é justificada pela maior ocorrência das lesões de alto grau. 0 intervalo entre os exames deve ser de no mínimo três anos, após dois exames negativos com intervalos de um ano ${ }^{5}$.

Frente aos fatores de risco para o desenvolvimento da doença, o principal deles é o Papilomavírus Humano (HPV), particularmente, os de alto risco oncogênico, que podem ser detectados em 99,7\% dos casos confirmados. Além disso, existem outras condições que podem aumentar a incidência do câncer de colo uterino, tais como: 
Conhecimentos, atitudes e práticas..

início de atividade sexual com pouca idade, imunossupressão, multiparidade, tabagismo e uso prolongado de contraceptivos orais 6 .

Nesse sentido, torna de extrema importância o papel do profissional de saúde, frente a ações de prevenção da doença, uma vez que esse profissional, deve agir como facilitador para promover a realização do exame e incentivar sua prática, bem como demostrar sua importância, através da identificação e minimização dos fatores que podem servir de barreira para a sua não realização ${ }^{8}$. Compreendendo importância das ações de prevenção ao câncer do colo uterino e do acesso à sua realização, bem como a identificação do conhecimento das mulheres frente essa a essa temática, o objetivo deste estudo foi identificar conhecimentos, atitudes e práticas de mulheres frente ao exame preventivo do câncer do colo uterino.

\section{MÉTODO}

Trata-se de um estudo transversal, desenvolvido junto a 160 mulheres atendidas em uma unidade de saúde do Acre. Foi realizado em uma unidade de saúde da família (USF) de Rio Branco (AC), Brasil, composta por equipe multidisciplinar completa e que possui uma ampla área de abrangência com cerca de 1.500 famílias residentes na região. A escolha do local ocorreu, pois trata-se de unidade escola de referência para a instrumentalização da prática de acadêmicos.

Rio Branco (latitude: $9^{\circ} 58^{\prime 2} 26^{\prime \prime S}$; longitude: $67^{\circ} 48^{\prime} 27^{\prime \prime}$ ) está localizado na Amazônia Sul Ocidental, possui uma área territorial de 8.834,942 $\mathrm{km}^{2}$, densidade demográfica e população estimada em 38,03 hab/km e 401.155 habitantes, respectivamente. As mulheres representam $51,3 \%$ desta população\%.

Para o cálculo amostral foi considerado média mensal de mulheres atendidas na referida unidade no ano de 2017 cujo total de atendimentos no ano foi de 1.788 mulheres, sendo que 0 
Conhecimentos, atitudes e práticas..

intervalo de confiança foi de $95 \%$ e um percentual de margem de erro de $5 \%$, o que determinou uma amostragem mínima de 160 mulheres convidadas para participarem do estudo. Selecionou-se as mulheres por conveniência, atendidas na unidade investigada, no período de dezembro de 2018 e janeiro de 2019 que foi o período disponível pelos pesquisadores para a coleta de dados após a aprovação no comitê de ética em pesquisa. Todas as mulheres convidadas aceitaram participar do estudo e não houveram perdas de participantes.

Foram incluídas no estudo mulheres com idade igual ou superior a 18 anos, com vida sexual ativa e que frequentavam a unidade de saúde. Foram excluídas mulheres que frequentavam a unidade, mas que não residiam no município.

A coleta de dados foi realizada na própria unidade de saúde no momento da procura por atendimento, por meio da aplicação de um questionário semiestruturado com perguntas sobre as características sociodemográficas, comportamentais e o conhecimento, atitude e prática das mulheres sobre a realização do exame de Papanicolau. A duração da entrevista foi de 20 minutos, realizada em sala climatizada e previamente reservada para essa finalidade, sendo um local tranquilo e longe da interferência de outras pessoas.

Para a digitação, revisão e análise dos dados para as variáveis categóricas, foi utilizado o programa Statical Package for the Sciences (SPSS), na versão 21.0, sendo realizada a análise descritiva com estudo de frequência $e$ percentagem desses dados.

Para avaliar 0 conhecimento, atitudes e prática foi utilizado o método criado por Brenna e colaboradores $^{10}$, que consiste nas seguintes definições: a) conhecimento adequado: quando a mulher já tinha ouvido falar do exame e tinha conhecimento da sua indicação; b) conhecimento inadequado: quando nunca tinha ouvido falar do exame ou já tinha ouvido, mas não sabia qual a sua finalidade; c) atitude adequada: quando acreditava que fazer o exame 
Conhecimentos, atitudes e práticas...

periodicamente era necessário e o realizava para prevenir o câncer do colo do útero; d) atitude inadequada: quando a mulher acreditava que fazer o exame era necessário, mas não o realizava para prevenção do câncer cervical; e) prática adequada: quando ela havia realizado seu último exame no máximo há três anos; d) prática inadequada: quando a mesma havia realizado o último exame há mais de três anos, realizava 0 exame raramente ou nunca havia feito .

A pesquisa foi submetida ao Comitê de Ética e Pesquisa do Centro Universitário UNINORTE e aprovada com o parecer número 3.000.295 e CAAE 97746818.3.0000.8028 e ao Comitê de Ética e Pesquisa do Hospital das Clínicas do Acre - HCA/FUNDACRE, aprovada com o parecer número 3.054.092 e CAAE 97746818.3.3001.5009.

\section{RESULTADOS E DISCUSSÃO}

Tabela 1 - Características sociodemográficas de mulheres atendidas em uma unidade de saúde. 2018-2019. Rio Branco - AC, Brasil. $(n=160)$

\begin{tabular}{|c|c|c|}
\hline Variável & $\mathbf{n}$ & $\%$ \\
\hline \multicolumn{3}{|l|}{ Faixa etária (anos) } \\
\hline $18-24$ & 26 & 16,3 \\
\hline $25-29$ & 22 & 13,8 \\
\hline $30-40$ & 55 & 34,4 \\
\hline $41-50$ & 28 & 17,5 \\
\hline $51-60$ & 18 & 11,3 \\
\hline$>60$ & 11 & 6,7 \\
\hline \multicolumn{3}{|l|}{ Escolaridade } \\
\hline Não alfabetizada & 1 & 0,6 \\
\hline Ensino Fundamental & 38 & 23,7 \\
\hline Ensino Médio & 74 & 46,3 \\
\hline Ensino Superior & 31 & 19,4 \\
\hline Pós-Graduação & 16 & 10,0 \\
\hline \multicolumn{3}{|l|}{ Situação conjugal } \\
\hline Com companheiro & 109 & 68,1 \\
\hline Sem companheiro & 51 & 31,9 \\
\hline \multicolumn{3}{|c|}{ Renda familiar (mensal)* } \\
\hline Sem renda & 27 & 16,9 \\
\hline$<1 S M$ & 25 & 15,6 \\
\hline $1 \mathrm{SM}$ & 53 & 33,1 \\
\hline $2-3 \mathrm{SM}$ & 43 & 26,9 \\
\hline$>4 \mathrm{SM}$ & 12 & 7,5 \\
\hline Total & 160 & 100,0 \\
\hline
\end{tabular}

Nota: *Valor do salário mínimo (SM) = R\$ 954,00. 
$\mathrm{Na}$ Tabela 1, concernente à faixa etária, a maioria das mulheres encontrava-se entre 30 a 40 anos $55(34,4 \%)$, achado que corrobora com estudo realizado em São Mateus $(\mathrm{ES})^{11}$ em unidades básicas de saúde, que identificou que a maior parte das mulheres estava na faixa etária de 25 a 44 anos $(67,78 \%)$. Resultado similar foi encontrado por um estudo realizado em Pernambuco (BR), que avaliando o perfil de mulheres com câncer de colo do útero atendidas para tratamento em um centro de oncologia, evidenciou uma média de idade de 40 a 59 anos entre as mesmas $^{12}$.

No que diz respeito ao grau de escolaridade, $75(46,3 \%)$ das participantes relataram ter ensino médio, no entanto em pesquisa realizada em Serranópolis de Minas $(M G)^{13} \quad$ sobre 0 perfil socioeconômico e prática do exame de prevenção de colo do útero de mulheres, constatou-se que a maioria $(45,4 \%)$ possuíam o ensino fundamental incompleto.

A baixa escolaridade é um fator relevante frente as ações de promoção e prevenção à saúde, pois limita a adesão das mulheres as ações e medidas preventivas e dificulta 0 entendimento das mesmas sobre a importância do exame $^{14}$. Um estudo realizado com mulheres na Índia, China, México, Rússia e África do Sul ao analisar o status econômico e educacional ao longo do seu ciclo da vida, em relação ao rastreamento do câncer de colo do útero, evidenciou que as mulheres com maior grau de instrução, apresentaram 10 vezes mais probabilidade de participar dos programas de triagem do câncer de colo do útero, em detrimento das demais mulheres ${ }^{15}$.

No que se refere à situação conjugal, $109(68,1 \%)$ das mulheres referiam ter um companheiro. A predominância de mulheres casadas, está em concordância com um estudo sobre o conhecimento, atitudes e prática sobre a triagem e prevenção do câncer do colo do útero entre mulheres de Taraba, nordeste da Nigéria, que evidenciou em seu estudo que a maioria das mulheres também eram casadas ${ }^{16}$.

No que tange a renda familiar mensal, 53 (33,1\%) afirmaram que recebiam um salário mínimo. De forma semelhante, estudo capixaba ${ }^{11}$ evidenciou-se 
Conhecimentos, atitudes e práticas...

que a maioria das mulheres (73,33\%) apresentava renda familiar de 1 a 2 salários mínimos. Semelhantemente em uma pesquisa realizada em Minas Gerais $(B R)^{13}$, constatou-se que a maioria das mulheres entrevistadas recebiam em média 1 salário mínimo por mês $(81,8 \%)$.
Os achados de um estudo realizado no Ambulatório de Ginecologia Oncológica do Hospital das Clínicas da Faculdade de Medicina de Botucatu (SP), considerou que renda familiar precária, associada a outros fatores, foi predominante $\mathrm{e}$ contribuiu para a vulnerabilidade à neoplasia ${ }^{17}$.

Tabela 2 - Características comportamentais de mulheres atendidas em uma unidade de saúde. 2018-2019. Rio Branco - AC, Brasil. $(n=160)$

\begin{tabular}{lcc}
\hline Variável & $\mathbf{n}$ & $\%$ \\
\hline Tabagista & 20 & 12,5 \\
Sim & 140 & 87,5 \\
Não & & \\
Etilista & 41 & 25,6 \\
Sim & 119 & 74,4 \\
Não & & \\
Iniciação sexual (idade) & 43 & 26,9 \\
$<15$ anos & 117 & 73,1 \\
$>15$ anos & & 16,2 \\
Infecções sexualmente transmissíveis & 26 & 83,8 \\
Sim & 134 & $\mathbf{1 0 0 , 0}$ \\
Não & 160 & \\
\hline Total & &
\end{tabular}

No que diz respeito às características comportamentais, $140(87,5 \%)$ afirmaram não ser fumantes. Da mesma forma, estudo realizado em Formosa da Serra Negra (MA), 90,4\% das participantes também não possuíam o hábito de fumar ${ }^{18}$, assim como estudo realizado em Teresina $(\mathrm{PI})^{19}$, onde $96,1 \%$ das mulheres negaram esta prática.
Segundo as diretrizes do rastreamento do câncer do colo do útero $^{20}$, o tabagismo está diretamente associado ao aparecimento dessa neoplasia, uma vez que mulheres que fumam têm o dobro de probabilidade de desenvolver câncer de colo do útero em relação àquelas que não fumam, pois, o hábito de fumar torna as defesas do sistema imunológico menos eficazes. 
Conhecimentos, atitudes e práticas..

Com relação ao consumo de bebida alcoólica, 119 (74,4\%) das mulheres negaram o uso. De acordo com o Instituto Nacional de Câncer (INCA), estudos mostram que consumir álcool aumenta o risco de desenvolver diferentes tipos de câncer ${ }^{21}$. Nesse sentido, o fato de a maioria das mulheres não fazerem dessas substâncias, configura-se como fator de proteção contra o aparecimento da doença e como medida que beneficia a saúde.

Um estudo realizado no Departamento de Radioterapia Oncológica da universidade da Califórnia (EUA), junto a 97 mulheres, identificou a associação de que o uso excessivo de álcool aumenta o risco de adquirir a doença e aumenta a mortalidade por câncer do colo do útero ${ }^{22}$.

No que se refere ao início da vida sexual, $117(73,1 \%)$ das participantes relataram que iniciaram a vida sexual após os 15 anos de idade. Esse resultado diverge do estudo realizado pelo INCA sobre a caracterização de mulheres com câncer cervical atendidas no Inca por tipo histológico, onde 40,7\%, haviam iniciado atividade sexual antes dos 16 anos de idade ${ }^{23}$.

Concernente às infecções sexualmente transmissíveis (IST), evidenciou-se que 134(83,8\%) relataram que até então nunca tinham contraído nenhuma delas, resultado que pode ser considerado como positivo, pois atualmente alguns estudos epidemiológicos ambos realizados na Itália, vem demostrando que IST estão frequentemente presentes em mulheres infectadas pelo HPV, sendo que essa é uma das infecções mais prevalentes entre mulheres com menos de 35 anos em todo o mundo e que está associada ao aparecimento do câncer de colo uterino ${ }^{24,25}$.

Outrossim, estudo realizado em um centro de atendimento terciário na Grécia, em 345 mulheres que realizaram o exame preventivo do câncer do colo uterino, evidenciou que entre as mulheres como resultado positivo para o câncer, o HPV foi detectado em 61 mulheres $(17,7 \%)$ e IST em $82(23,8 \%)^{26}$. 
Tabela 3 - Conhecimento, atitudes e prática frente o exame preventivo do câncer do colo uterino entre de mulheres atendidas em uma unidade de saúde. 2018-2019. Rio Branco AC, Brasil. $(n=160)$

\begin{tabular}{lcc}
\hline Variável & $\mathbf{n}$ & $\%$ \\
\hline Conhecimento & & \\
Adequado & 85 & 53,1 \\
Inadequado & 75 & 46,9 \\
Atitude & & \\
Adequada & 156 & 97,5 \\
Inadequada & 04 & 2,5 \\
Prática & & 58,8 \\
Adequada & 94 & 41,2 \\
Inadequada & 66 & 100,0 \\
\hline Total & 160 & \\
\hline
\end{tabular}

Na Tabela 3, observa-se que $85(53,1 \%)$ das participantes apresentaram conhecimento adequado sobre a doença, 156 $(97,5 \%)$ possuíam atitude adequada, pois achavam importante a realização do exame preventivo do câncer do colo uterino e 94 (58,8\%) demostrarem prática adequada, pois haviam realizado exame nos últimos 3 anos.

Esses achados corroboram com estudo que também buscou evidenciar o conhecimento, atitudes e práticas de mulheres no Zimbábue na região da África Subsaariana sobre câncer cervical e HPV, métodos atuais de triagem e vacinação ${ }^{27}$, onde identificou-se que as mesmas demostram ter (CAP) adequadas sobre o exame, mas não tinham conhecimento frente aos fatores de risco para a o desenvolvimento da doença. De forma divergente, os achados de um estudo de revisão da literatura em artigos nacionais e internacionais frente a essa temática, identificou-se que até então em muitos locais, o conhecimento das mulheres acerca do exame preventivo é superficial e com pouca clareza do significado da prevenção, enfatizando a necessidade de intensificação das ações de educação em saúde ${ }^{28}$. Diante disso, torna-se cada vez mais importante, a implementação de práticas educativas inovadores, voltadas para a valorização das mulheres, como transformadoras da realidade a sua volta, sensibilizando-as para o autocuidado e para a capacidade do desenvolvimento da autonomia, uma vez que as intervenções de educação em saúde são úteis para melhorar o conhecimento e 
Conhecimentos, atitudes e práticas...

percepções das mulheres sobre seu corpo e os riscos inerente ao câncer ${ }^{29-33}$.

Dentre as limitações do presente estudo, estão inevitavelmente os vieses de memória e de informação, característica desse tipo de estudo.

\section{CONCLUSÃO}

Verificou-se que as participantes sabem da existência do exame, sua finalidade e importância, além de realiza-lo periodicamente. Os achados indicam que a intensificação das ações de educação em saúde frente a esse público alvo, continuam sendo essenciais, a fim de fortalecer no cotidiano destas mulheres o processo de autonomia e busca de ações promotoras de saúde.

Diante do exposto, destacase o papel do profissional de saúde enquanto educador, podendo influenciar os comportamentos, atitudes e práticas da população, principalmente em relação ao exame de Papanicolau e outros que integram a saúde da mulher.

\section{REFERÊNCIAS}

1. Figueiredo MC, Melo Júnior JM, Segati KD. Prevalência de lesões precursoras para o câncer de colo de útero nas regiões e sua relação com a cobertura do programa de rastreamento. Femina. 2014; 42(6):295-302.

2. Bray F, Ferlay J, Soerjomataram I, Siegel RL, Torre LA, Jemal A. Global Cancer Statistics 2018: Globocan estimates of incidence and mortality worldwide for 36 cancers in 185 countries. CA Cancer J Clin. 2018; 68(6):394424.

3. Ministério da Saúde (BR). Instituto Nacional de Câncer José Alencar Gomes da

Silva (INCA). Estimativa 2018: incidência de câncer no Brasil. Brasília (DF): Ministério da Saúde; 2017.

4. Aguilar RP, Soares DA. Barreiras à realização do exame Papanicolau: perspectivas de usuárias e profissionais da estratégia de saúde da família da cidade de Vitória da Conquista BA. Physis. 2015; 25(2):359-379.

5. Organização Mundial da Saúde (OMS). Nota de orientação da 
Conhecimentos, atitudes e práticas..

OPAS/OMS: prevenção e controle de amplo alcance do câncer do colo do útero: um futuro mais saudável para meninas e mulheres. Washington, DC: OPAS, 2013.

6. Mirzaie-Kashani E, Bouzari M, Talebi A, Arbabzadeh-Zavareh F. Detection of Human Papillomavirus in Chronic Cervicitis, Cervical Adenocarcinoma, Intraepithelial Neoplasia and Squamus Cell Carcinoma. Jundishapur J Microbiol 2014; 7(5):1-5.

7. Stewart B, Wild C. World cancer report. 1st ed. Lyon: IARC press; 2014.

8. Costa RSL, Rodrigues MV, Souza TN. Fatores que levam a não adesão ao exame preventivo do câncer do colo uterino em uma unidade de saúde do Acre em 2014. DêCiência em Foco. 2018; 2(2):5-18.

9. Instituto Brasileiro de Geografia e Estatística (IBGE). Resultados do Censo 2010: Rio Branco. Rio de Janeiro: IBGE; 2012.

10. Brenna SMF, Hardy E, Zeferino LC, Namura I. Conhecimento, atitude e prática do exame de Papanicolaou em mulheres com câncer de colo uterino. Cad Saúde Pública. 2001; 17(4):90914.

11. Sena LX, Souza NA, Gradella DBT. Conhecimento, atitude e prática do exame Papanicolau por mulheres do norte do Espírito Santo. Enc Biosfera. 2018; 15(27):102-12.

12. Silva RCG, Silva ACO, Peres AL, Oliveira SR. Profile of women with cervical cancer attended for treatment in oncology center. Rev Bras Saude Mater Infant. 2018; 18(4):695-702.

13. Dias EG, Santos DDC, Dias ENF, Alves JCS, Soares LR. Perfil socioeconômico e prática do exame de prevenção do câncer do colo do útero de mulheres de uma unidade de saúde. Rev Saude e Desen. 2015; 7(4):135146.

14. Silva MAS, Teixeira EMB, Ferreira RAP, Cestari MEW, Cardelli AAM. Fatores relacionados a não adesão à realização do exame de Papanicolau. Rev Rene. 2015; 16(4):532-539.

15. Akinyemiju T, Ogunsina $\mathrm{K}$, Sakhuja S, Ogbhodo V, Braithwaite D. Life-course 
Conhecimentos, atitudes e práticas...

socioeconomic status and breast and cervical cancer screening: analysis of the WHO's Study on Global Ageing and Adult Health (SAGE). BMJ Open. 2016; 6:1-10.

16. Rimande-Joel R, Ekenedo G. Knowledge, Belief and Practice of Cervical Cancer Screening and Prevention among Women of Taraba, North-East Nigeria. Asian Pac J Cancer P. 2019; 20(11):3291-3298.

17. Conde CR, Lemos TMR, Ferreira MLSM.

Características sociodemográficas, individuales y de programación de las mujeres con cáncer cervical. Enferm glob. 2018; 17(49):348380.

18. Lopes NC, Aragão FBA, Pereira JFS, Oliveira KCC, Boas DP, Silva EC et al. Exame de colpocitologia oncótica: conhecimento e prática de usuárias de uma unidade básica de saúde em Formosa da Serra Negra - MA. Braz J Surg Clin. 2017; 20(1):49-53.

19. Oliveira AC, Pessoa RS, Carvalho AMC, Magalhães RLB. Fatores de risco e proteção à saúde de mulheres para prevenção do câncer uterino. Rev Rene. 2014; 15(2):240-48.

20. Ministério da Saúde (BR). Instituto Nacional de Câncer José Alencar Gomes da Silva (INCA). Diretrizes brasileiras para o rastreamento do câncer do colo do útero. Brasília (DF): Ministério da Saúde; 2016.

21. Ministério da Saúde (BR). Instituto Nacional de Câncer José Alencar Gomes da Silva (INCA). Causas e Prevenção: Bebidas Alcoólicas. Brasília (DF): Ministério da Saúde; 2019.

22. Mayadev J, Li CS, Lim J, Valicenti R, Alvarez EA. Alcohol abuse decreases pelvic control and survival in cervical cancer: an opportunity for lifestyle intervention to improve outcomes. Am J Clin Oncol. 2017; 40(5):451-457.

23. Rozario S, Silva IF, Koifman RJ, Silva IF. Caracterização de mulheres com câncer cervical atendidas no Inca por tipo histológico. Rev Saúde Pública. 2019; 53:88.

24. Bellaminutti $S$, Seraceni $S$, De Seta F, Gheit T, Tommasino M, Comar M. HPV and Chlamydia trachomatis co-detection in 
Conhecimentos, atitudes e práticas...

young asymptomatic women from high incidence area for cervical cancer. Med Virol. 2014; 86(11):1920-1925.

25. Camporiondo MP, Farchi F, Ciccozzi M, Denaro A, Gallone D, Maracchioni $F$, et al. Detection of HPV and coinfecting pathogens in healthy Italian women by multiplex real-time PCR. Infez Med. 2016; 24(1):12-17.

26. Parthenis C, Panagopoulos $P$, Margari N, Kottaridi C, Spathis A, Pouliakis A, et al. The association between sexually transmitted infections, human papillomavirus, and cervical cytology abnormalities among women in Greece. Int J Infect Dis. 2018; 73:72-77.

27. Mapanga W, Girdler-Brown B, Singh E. Knowledge, attitudes and practices of young people in Zimbabwe about cervical cancer and HPV, current methods of screening and vaccination. BMC Cancer. 2019; 19(1):1-14.

28. Silva BL, Santos RNLC, Ribeiro FF, Anjos UU, Ribeiro KSQS. Prevenção do câncer de colo uterino e ampliação da faixa etária de risco. Rev enferm UFPE online. $2014 ; 8(6)$ :148290.

29. Vasconcellos SA, Muniz RM, Viegas AC, Cardoso DH, Amaral DED, Barboza MCN. Mulher com câncer do colo do útero: trajetória terapêutica. J Health NPEPS. 2016; 1(1):17-30.

30. Zeledón PIZ, Turcios MCL. Conocimientos y factores culturales de mujeres que intervienen en la realización del papanicolaou en Nicaragua. J Health NPEPS. 2017; 2(1):206217.

31. Silva RGM, Nascimento VF, Santos POF, Ferreira MZJ. Teste de Papanicolau: realização e conhecimento de acadêmicas de enfermagem. Rev epidemiol controle infecç. 2019; 9(1):8186.

32. Souza KR, Paixão GPN, Almeida ES, Souza AR, Lírio JGS, Campos LM. Educação popular como instrumento participativo para a prevenção do câncer ginecológico: percepção de mulheres. Rev Cuid. 2015; 6(1):892-899.

33. Ebu NI, Amissah-Essel S, Asiedu C, Akaba S, Pereko KA. Impact 


$\begin{array}{ll}\text { of } \quad \text { health } \quad \text { education } & \text { screening for women in Ghana. } \\ \text { intervention on cervical cancer } & \text { BMC public health. 2019; } \\ \text { knowledge and perception and } & \text { 19(1505):1-11. }\end{array}$

Conflito de interesses: Os autores declaram não haver conflito de interesses.

\section{Participação dos autores:}

- Concepção: Mesquita AD, Teles KKN, Silva SCB, Silva FR, Lima LKC, Costa RSL, Arruda EF.

- Desenvolvimento: Mesquita AD, Teles KKN, Silva SCB, Costa RSL, Arruda EF.

- Redação e revisão: Mesquita AD, Teles KKN, Silva SCB, Costa RSL, Arruda EF.

Como citar este artigo: Mesquita $A D$, Teles KKN, Silva SCB, Silva FR, Lima LKC, Costa RSL, et al. Conhecimentos, atitudes e práticas de mulheres frente ao exame preventivo do câncer do colo uterino. J Health NPEPS. 2020; 5(1):261275.

Submissão: $21 / 11 / 2019$

Aceito: $25 / 03 / 2020$

Publicado: $01 / 06 / 2020$ 